\title{
Atherosclerosis in man: natural history and effects
}

\author{
By G. A. Gresham, Department of Pathology, University of Cambridge
}

The term natural history is often used as an excuse for vague discursive accounts of biological matters; nothing could be less desirable in a subject like atherosclerosis where confusions of definition have for long hindered progress. I propose to try and present a concise and precise account of the natural history and effects of this disease in man and to indicate some of the main debatable points about the human disease.

Atherosclerosis is found in a variety of animals such as birds, pigs, and nonhuman primates but it is more in the nature of an incidental finding rather than a serious disease. In man it provides a main cause of death in affluent western communities and it produces these effects by vascular obstruction which is either due to the disease itself or to thrombosis of vessels already affected by atherosclerosis. There is still debate about the relative importance of these two occluding factors as a cause of human disease. It is primarily a disease of large and medium-sized arteries such as the aorta and its principal branches and, in particular, the coronary arteries. Cerebral vessels are also involved. Only very rarely is it seen in veins. It is a progressive disease throughout life and has been found even in stillborn foetuses.

The first indications appear in the first decade of life as the so-called fatty streaks or spots. They are most often seen in the proximal aorta, that is the ascending part of the arch, and in the thoracic segment. In later life fibrous plaques and lesions laden with lipid (atheroma) appear but the site of predilection is now the distal aorta in the abdomen (Fig. I). This profound difference in distribution of early and later lesions has led to some controversy about the significance of the fatty streak. Some say that it is not the early stage of atherosclerosis, others do not agree. It is a

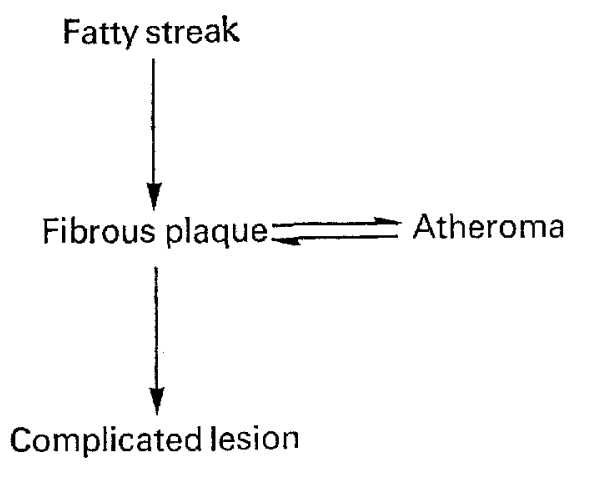

Fig. I. The evolution of the atherosclerotic lesion. 
fundamental point because most of the lesions that experimentalists examine in animals are predominantly of the fatty-streak type. Fatty streaks do occur in the proximal internal carotid, in the carotid sinus region at an early age and never disappear but progress slowly to more advanced atherosclerotic lesions. So it does not always follow that the fatty streak is not the precursor. Transitions between fatty streaks and more advanced lesions can be found in the aorta; so again there is some relationship. Finally, from experimental work we do know that fatty streaks are evanescent lesions that can be made to disappear in rabbits after withdrawal of a high-cholesterol diet.

Most people agree that the atherosclerotic lesion is primarily an intimal disorder (Pl. I). There is still disagreement about the earlier steps in the process. Views range from intimal oedema, lipid or mucopolysaccharide accumulation and so on. These early stages are at present being studied by electron microscopy and other methods. The earliest stage revealed by the light microscope is a swelling of the subendothelial tissues followed by an accumulation of cells, fibres, and substances such as lipids and mucopolysaccharide. This is the structure of the fatty streak. Cells that appear are of smooth-muscle type, and the fibres are collagen, elastin, and reticulin. The later stage of fibrous plaque is characterized by the appearance of a lot of collagen as a cap in the intima. A variant of this, the atheroma, is also replete with lipid. This is an important lesion because it provides the main reason for the occlusive effect of atherosclerosis. Later still the lesion is complicated by superimposed thrombosis, haemorrhage into the plaque, underlying ischaemic fibrosis, and calcification of the media.

The effects of atherosclerosis are closely related to the histopathological sequence that I have described. The vessels involved are large ones such as the aorta and its main branches. The distribution of lesions varies from one individual to another. The degree of disease in the aorta is usually paralleled by that in the coronary vessels but this is not always so, particularly in hyperlipidaemic subjects. Involvement of the cerebral vessels by atherosclerosis is almost uniquely associated with hypertension and atherosclerotic disease of branches of the circle of Willis accounts for rupture of intracranial vessels into the brain or its surroundings.

Broadly speaking, the effects of atherosclerosis are the result of three events: atherosclerotic narrowing, superimposed thrombosis, and weakening of the media with aneurysm formation.

There is still much discussion about the relative importance of atherosclerosis itself and of atherosclerosis with thrombosis as the cause of the present epidemic of ischaemic heart disease. In many of the sudden unexpected deaths the principal feature is occlusive atherosclerosis of the coronary artery and the finding of recent or old myocardial infarction is by no means frequent. Often, the heart muscle appears, macroscopically and microscopically, normal and one is left wondering why acute ventricular failure should have occurred when it did. Thrombi in diseased coronary arteries are seen most often in association with visible evidence of myocardial damage either recent or old and the patient may frequently have suffered symptoms of cardiac ischaemia. 


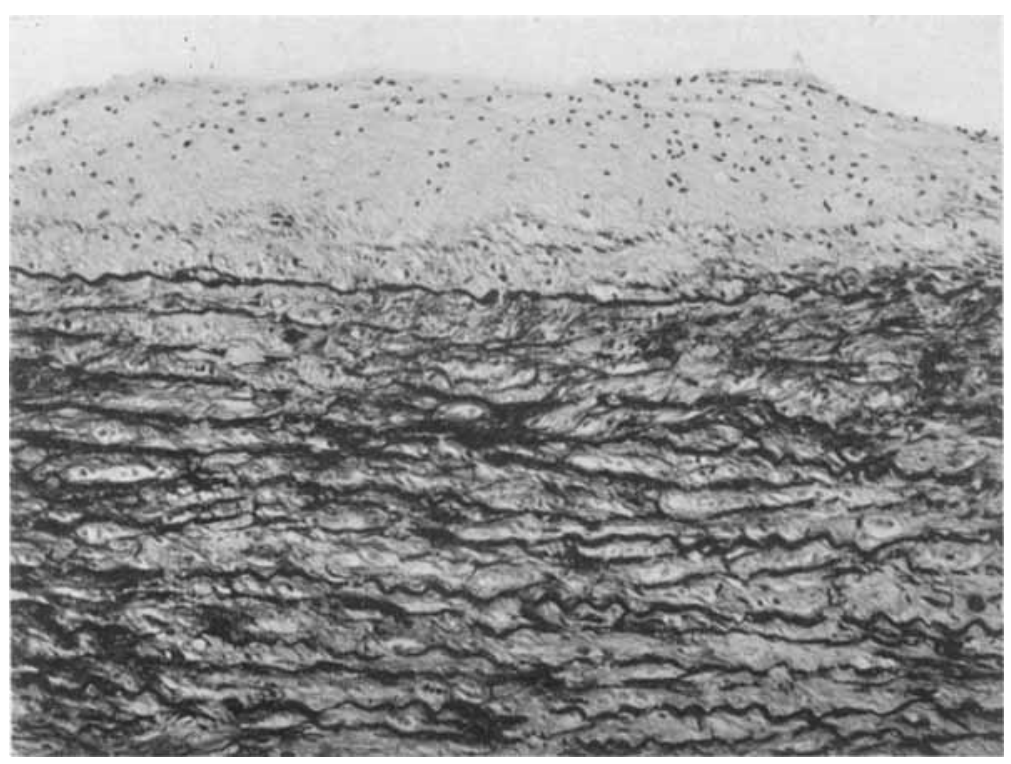

G. A. GRESHAM

(Facing p. 305) 
Weakening of the vessel wall in association with atherosclerosis is still a mysterious process. Most often it affects the distal aorta or the cerebral and other vessels leading to medial fibrosis, and abnormal dilatation of the aorta which may burst. It is probable that mural ischaemia caused by impeded intimal diffusion of nutrients is an important factor in the pathogenesis of this.

Atherosclerotic occlusion of vessels other than the coronary arteries is common and provides a variety of syndromes according to the vessels that are involved. Occlusion of limb vessels produces claudication. Narrowing of the renal arteries producing renal ischaemia can of itself cause hypertension. Blockage of mesenteric vessels causes abdominal angina and either necrosis or fibrosis of the gut. Not infrequently several manifestations of atherosclerotic occlusion are to be found in the same patient.

Fragments of thrombus, cholesterol and other material may detach from the surface of complicated atherosclerotic plaques in the aorta or in the great vessels producing a galaxy of effects varying from retinal ischaemia to gangrene of the toes.

Most of the complications of atherosclerosis appear when the disease is advanced and there is little hope of discovering the aetiology of the disease from a study of this stage of the disease. Retrospective studies of patients with complications of atherosclerosis reveal certain well-defined risk factors: diabetes, hyperlipidaemia, hypertension, cigarette smoking, and others less well defined such as obesity. The relation to diet of animal fats or to excessive intake of other substances, like sucrose, is still a hotly debated subject. Racial and other studies cast some light on the matter but the doubt of accuracy of diagnosis always remains as a serious obstacle in work of this kind.

Experimental work in animals is concerned mainly with the induction of changes in blood and arterial wall lipids, and with the formation of fatty streaks. Problems of species differences, grading the extent of disease, and undue preoccupation with the aorta rather than the coronary arteries all make interpretation and extrapolation to man difficult.

Like cancer, we know much of atherogenesis. Which particular factor is mainly responsible for this modern epidemic is still unsolved.

EXPLANATION OF PLATE

Pale-staining, intimal thickening seen in the fatty streak. Haematoxylin and eosin, $\times 100$. 